\title{
The induced generalized OWA operator
}

\author{
José M. Merigó*, Anna M. Gil-Lafuente \\ Department of Business Administration, University of Barcelona, Av. Diagonal 690, 08034 Barcelona, Spain
}

\section{A R T I C L E I N F O}

\section{Article history:}

Received 25 March 2008

Received in revised form 10 November 2008

Accepted 12 November 2008

\section{Keywords:}

Aggregation operator

OWA operator

Generalized mean

Quasi-arithmetic mean

Decision-making

\begin{abstract}
A B S T R A C T
We present the induced generalized ordered weighted averaging (IGOWA) operator. It is a new aggregation operator that generalizes the OWA operator, including the main characteristics of both the generalized OWA and the induced OWA operator. This operator uses generalized means and order-inducing variables in the reordering process. It provides a very general formulation that includes as special cases a wide range of aggregation operators, including all the particular cases of the IOWA and the GOWA operator, the induced ordered weighted geometric (IOWG) operator and the induced ordered weighted quadratic averaging (IOWQA) operator. We further generalize the IGOWA operator via quasi-arithmetic means. The result is the Quasi-IOWA operator. Finally, we present a numerical example to illustrate the new approach in a financial decision-making problem.
\end{abstract}

(c) 2008 Elsevier Inc. All rights reserved.

\section{Introduction}

A wide range of aggregation operators are found in the literature. One common aggregation method is the ordered weighted averaging (OWA) operator [29]. It provides a parameterized family of aggregation operators that include as special cases the maximum, the minimum and the average. Since its appearance, the OWA operator has been used in a wide range of applications [1-8,11-45].

In [43], Yager and Filev, motivated by the work of Mitchell and Estrakh [20], developed an extension of the OWA operator, called the induced ordered weighted averaging (IOWA) operator. The difference is that the reordering step is no longer determined only by the values of the arguments, but could be induced by another mechanism, such that the ordered position of the arguments; in other words, the reordering can depend on the values of their associated order-inducing variables. In the last few years, the IOWA operator has received increasing attention, e.g., [7,8,12,13,28,34,35,37].

Another interesting extension is the generalized OWA (GOWA) operator $[15,38]$, which uses generalized means [9,10] in the OWA operator. It generalizes a wide range of mean operators such as the arithmetic mean (AM), the geometric mean $(\mathrm{GM})$, the quadratic mean (QM), the OWA operator, the ordered weighted geometric (OWG) operator and the ordered weighted quadratic averaging (OWQA) operator. In [3], Beliakov developed a further extension of the GOWA operator, and obtained the Quasi-OWA operator introduced by [11]. Further studies on these generalizations are found in [4,5].

The aim of this paper is to present the induced generalized OWA (IGOWA) operator. It is an extension of the OWA operator that uses the main characteristics of both the IOWA and the GOWA operator. That is to say, it uses order-inducing variables in the reordering process and generalized means. Then, we can obtain a generalization that includes the IOWA operator and its particular cases, as well as many other situations, such as the induced OWG (IOWG) operator [7,28], the induced OWQA (IOWQA) operator and the induced ordered weighted harmonic averaging (IOWHA) operator. This generalization also includes the GOWA operator and its special cases such as the OWA, the generalized mean (GM), the weighted generalized

\footnotetext{
* Corresponding author. Tel.: +34 9340219 62; fax: +349340398 82 .

E-mail addresses: jmerigo@ub.edu (J.M. Merigó), amgil@ub.edu (A.M. Gil-Lafuente).
} 
mean (WGM), etc. We study different properties and families of this operator such as the olympic-IGOWA, the median-IGOWA, etc.

We further generalize the IGOWA operator by using quasi-arithmetic means, obtaining the Quasi-IOWA operator. Note that the Quasi-IOWA can be seen as an extension of the Quasi-OWA operator that uses order-inducing variables in the reordering process. With this generalization, we get as special cases the original IGOWA operator as well as many other known operators such as the exponential IOWA, the trigonometric IOWA and the radical IOWA, among others.

We also present an application of the new approach in an example of investment selection. The main advantage of the IGOWA operator in decision-making is that it includes a lot of particular cases that can be used for making the decision. Therefore, it is possible to consider different types of aggregations that may lead to different decisions. Note that to a certain extent, the OWA operator has the same advantage, but with the IGOWA, we have more possibilities. The operator could also be used for other decision-making applications such as the selection of financial products, human resource management, strategic decision-making, product management, and others.

This paper is organized as follows. In Section 2, we briefly review some basic concepts such as the OWA, the IOWA and the GOWA operators. In Section 3, we present the IGOWA operator. Section 4 analyzes different families of IGOWA operators. In Section 5 we introduce the Quasi-IOWA operator. In Section 6, an application of the new approach is presented. Finally, Section 7 summarizes the main conclusions of the paper.

\section{Preliminaries}

In this section, we briefly describe the OWA operator, the IOWA operator and the GOWA operator.

\subsection{OWA operator}

The OWA operator was introduced by Yager in [29] and provides a parameterized family of aggregation operators that includes the arithmetic mean, the maximum and the minimum. It can be defined as follows.

Definition 1. An OWA operator of dimension $n$ is a mapping OWA: $R^{n} \rightarrow R$ defined by an associated weighting vector $W$ of dimension $n$, such that the sum of the weights is 1 and $w_{j} \in[0,1]$, according to the following formula:

$$
\operatorname{OWA}\left(a_{1}, a_{2}, \ldots, a_{n}\right)=\sum_{j=1}^{n} w_{j} b_{j}
$$

where $\left(b_{1}, b_{2}, \ldots, b_{n}\right)$ is simply $\left(a_{1}, a_{2}, \ldots, a_{n}\right)$ reordered from largest to smallest.

We can generalize the direction of the reordering, and distinguish between the descending OWA (DOWA) operator and the ascending OWA (AOWA) operator [30]. The OWA operator is commutative, monotonic, bounded and idempotent [29].

\subsection{IOWA operator}

The IOWA operator was introduced by Yager and Filev [43] and it represents an extension of the OWA operator. The main difference is that the reordering step of the IOWA is carried out with order-inducing variables, rather than depending on the values of the arguments $a_{i}$. The IOWA operator also includes the maximum, the minimum and the average operators, as special cases. It can be defined as follows:

Definition 2. An IOWA operator of dimension $n$ is a mapping IOWA: $R^{n} \rightarrow R$ defined by an associated weighting vector $W$ of dimension $n$ such that the sum of the weights is 1 and $w_{j} \in[0,1]$, and a set of order-inducing variables $u_{i}$, by a formula of the following form:

$$
\operatorname{IOWA}\left(\left\langle u_{1}, a_{1}\right\rangle,\left\langle u_{2}, a_{2}\right\rangle \ldots,\left\langle u_{n}, a_{n}\right\rangle\right)=\sum_{j=1}^{n} w_{j} b_{j}
$$

where $\left(b_{1}, \ldots, b_{n}\right)$ is simply $\left(a_{1}, a_{2}, \ldots, a_{n}\right)$ reordered in decreasing order of the values of the $u_{i}, u_{i}$ is the order-inducing variable and $a_{i}$ is the argument variable.

The IOWA operator is also monotonic, bounded, idempotent and commutative [43]. Other properties and particular cases of the IOWA operators are studied in [35,43].

\subsection{GOWA operator}

The generalized OWA (GOWA) operator was introduced in $[11,38]$. It uses generalized means in the OWA operator. It can be defined as follows: 
Definition 3. A GOWA operator of dimension $n$ is a mapping GOWA: $R^{n} \rightarrow R$ defined by an associated weighting vector $W$ of dimension $n$ such that the sum of the weights is 1 and $w_{j} \in[0,1]$, and a parameter $\lambda \in(-\infty, \infty)$, according to the following formula:

$$
\operatorname{GOWA}\left(a_{1}, a_{2}, \ldots, a_{n}\right)=\left(\sum_{j=1}^{n} w_{j} b_{j}^{\lambda}\right)^{1 / \lambda},
$$

where $\left(b_{1}, \ldots, b_{n}\right)$ is simply $\left(a_{1}, a_{2}, \ldots, a_{n}\right)$ reordered from largest to smallest.

In this case, it is also possible to distinguish between the descending generalized OWA (DGOWA) operator and the ascending generalized OWA (AGOWA) operator. The weights of these operators are related by $w_{j}=w_{n+1-j}^{*}$, where $w_{j}$ is the $j$ th weight of the DGOWA (or GOWA) operator and $w_{n+1-j}^{*}$ is the $j$ th weight of the AGOWA operator.

As it is explained in [11,38], the GOWA operator is commutative, monotonic, bounded and idempotent. It can also be demonstrated that the GOWA operator has as special cases the maximum, the minimum, the generalized mean and the weighted generalized mean. Note that the weighted generalized mean is obtained when $j=i$, for all $i$ and $j$, where $j$ is the $j$ th argument of the $b_{j}$ and $i$ is the $i$ th argument of the $a_{i}$.

By considering different values of the parameter $\lambda$, we can also obtain other special cases, including the usual OWA operator $(\lambda=1)$ [29], the ordered weighted geometric (OWG) operator $(\lambda=0)[6,27]$, the ordered weighted harmonic averaging (OWHA) operator $(\lambda=-1)$ [38] and the ordered weighted quadratic averaging (OWQA) operator $(\lambda=2)[38]$.

Another interesting issue to consider is the attitudinal character, which is defined by Yager in [38] as

$$
\alpha(W)=\left(\sum_{j=1}^{n} w_{j}\left(\frac{n-j}{n-1}\right)^{\lambda}\right)^{1 / \lambda}
$$

It can be shown that $\alpha \in[0,1]$. The more of the weight is concentrated near the top of $W$, the closer $\alpha$ approaches 1 , and the more of the weight is concentrated toward the bottom of $W$, the closer $\alpha$ approaches 0 . Note that for the optimistic criterion $\alpha(W)=1$ and for the pessimistic criterion $\alpha(W)=0$.

If we replace $b^{\lambda}$ with a general continuous strictly monotonic function $g(b)$ [3], then, the GOWA operator becomes the Quasi-OWA operator [11], which is defined as follows:

Definition 4. A Quasi-OWA operator of dimension $n$ is a mapping QOWA: $R^{n} \rightarrow R$ defined by an associated weighting vector $W$ of dimension $n$ such that the sum of the weights is 1 and $w_{j} \in[0,1]$, and a continuous strictly monotonic function $g(b)$, according to the following formula:

$$
\operatorname{QOWA}\left(a_{1}, a_{2}, \ldots, a_{n}\right)=g^{-1}\left(\sum_{j=1}^{n} w_{j} g\left(b_{(j)}\right)\right)
$$

where $\left(b_{1}, \ldots, b_{n}\right)$ is $\left(a_{1}, a_{2}, \ldots, a_{n}\right)$ reordered from largest to smallest.

\section{The induced generalized OWA operator}

The induced generalized OWA (IGOWA) operator is an extension of the GOWA operator, with the difference that the reordering step of the IGOWA operator is not defined by the values of the arguments $a_{i}$, but rather by order-inducing variables $u_{i}$, where the ordered position of the arguments $a_{i}$ depends upon the values of the $u_{i}$. Therefore, we get a more general formulation of the reordering process that it is able to consider more complex situations. It can be defined as follows:

Definition 5. An IGOWA operator of dimension $n$ is a mapping IGOWA: $R^{n} \rightarrow R$ defined by an associated weighting vector $W$ of dimension $n$ such that the sum of the weights is 1 and $w_{j} \in[0,1]$, a set of order-inducing variables $u_{i}$, and a parameter $\lambda \in(-\infty, \infty)$, according to the following formula:

$$
\operatorname{IGOWA}\left(\left\langle u_{1}, a_{1}\right\rangle,\left\langle u_{2}, a_{2}\right\rangle, \ldots,\left\langle u_{n}, a_{n}\right\rangle\right)=\left(\sum_{j=1}^{n} w_{j} b_{j}^{\lambda}\right)^{1 / \lambda}
$$

where $\left(b_{1}, \ldots, b_{n}\right)$ is $\left(a_{1}, a_{2}, \ldots, a_{n}\right)$ reordered in decreasing order of the values of the $u_{i}$, the $u_{i}$ are the order-inducing variables, and $a_{i}$ are the argument variables.

Example 1. Assume the following collection of arguments with their respective order-inducing variables $\left\langle u_{i}, a_{i}\right\rangle:\langle 7,25\rangle$, $\langle 2,40\rangle,\langle 10,20\rangle,\langle 3,60\rangle$. If we assume that $W=(0.2,0.2,0.3,0.3)$ and $\lambda=1$, then, the aggregation formula is

$$
0.2 \times 20+0.2 \times 25+0.3 \times 60+0.3 \times 40=39 .
$$


As we can see, the order-inducing variables $u_{i}$ reorder the argument variables $a_{i}$ in decreasing order.

Again, it is possible to distinguish the descending induced generalized OWA (DIGOWA) operator and the ascending induced generalized OWA (AIGOWA) operator. The weights of these operators are related by $w_{j}=w_{n+1-j}^{*}$, where $w_{j}$ is the $j$ th weight of the DIGOWA (or IGOWA) operator and $w_{n+1-j}^{*}$ the $j$ th weight of the AIGOWA operator.

If $B$ is the vector consisting of the ordered arguments $b_{j}^{\lambda}$, which we call the ordered argument vector and $W^{T}$ is the transpose of the weighting vector, then the IGOWA operator can be expressed as

$$
\operatorname{IGOWA}\left(\left\langle u_{1}, a_{1}\right\rangle,\left\langle u_{2}, a_{2}\right\rangle, \ldots,\left\langle u_{n}, a_{n}\right\rangle\right)=\left(W^{T} B\right)^{1 / \lambda} .
$$

Note that if the weighting vector is not normalized, i.e., $W=\sum_{j=1}^{n} w_{j} \neq 1$, then, the IGOWA operator can be expressed as

$$
\operatorname{IGOWA}\left(\left\langle u_{1}, a_{1}\right\rangle,\left\langle u_{2}, a_{2}\right\rangle, \ldots,\left\langle u_{n}, a_{n}\right\rangle\right)=\left(\frac{1}{W} \sum_{j=1}^{n} w_{j} b_{j}^{\lambda}\right)^{1 / \lambda} .
$$

The IGOWA operator is a mean or averaging operator. This is a reflection of the fact that the operator is commutative, monotonic, bounded and idempotent. These properties are proven in the following theorems:

Theorem 1. (Monotonicity): Let $f$ be the IGOWA operator. If $a_{i} \geqslant e_{i}$, for all $a_{i}$, then

$$
f\left(\left\langle u_{1}, a_{1}\right\rangle, \ldots,\left\langle u_{n}, a_{n}\right\rangle\right) \geqslant f\left(\left\langle u_{1}, e_{1}\right\rangle, \ldots,\left\langle u_{n}, e_{n}\right\rangle\right) .
$$

Proof. Let

$$
f\left(\left\langle u_{1}, a_{1}\right\rangle, \ldots,\left\langle u_{n}, a_{n}\right\rangle\right)=\left(\sum_{j=1}^{n} w_{j} b_{j}^{\lambda}\right)^{1 / \lambda}
$$

and

$$
f\left(\left\langle u_{1}, e_{1}\right\rangle, \ldots,\left\langle u_{n}, e_{n}\right\rangle\right)=\left(\sum_{j=1}^{n} w_{j} d_{j}^{\lambda}\right)^{1 / \lambda}
$$

Since $a_{i} \geqslant e_{i}$, for all $a_{i}$, it follows that, $a_{i} \geqslant e_{i}$, so

$$
f\left(\left\langle u_{1}, a_{1}\right\rangle, \ldots,\left\langle u_{n}, a_{n}\right\rangle\right) \geqslant f\left(\left\langle u_{1}, e_{1}\right\rangle, \ldots,\left\langle u_{n}, e_{n}\right\rangle\right) .
$$

Theorem 2. (Commutativity): Let $f$ be the IGOWA operator. Then

$$
f\left(\left\langle u_{1}, a_{1}\right\rangle, \ldots,\left\langle u_{n}, a_{n}\right\rangle\right)=f\left(\left\langle u_{1}, e_{1}\right\rangle, \ldots,\left\langle u_{n}, e_{n}\right\rangle\right),
$$

where $\left(\left\langle u_{1}, a_{1}\right\rangle, \ldots,\left\langle u_{n}, a_{n}\right\rangle\right)$ is any permutation of the arguments $\left(\left\langle u_{1}, e_{1}\right\rangle, \ldots,\left\langle u_{n}, e_{n}\right\rangle\right)$.

Proof. Let

$$
f\left(\left\langle u_{1}, a_{1}\right\rangle, \ldots,\left\langle u_{n}, a_{n}\right\rangle\right)=\left(\sum_{j=1}^{n} w_{j} b_{j}^{\lambda}\right)^{1 / \lambda}
$$

and

$$
f\left(\left\langle u_{1}, e_{1}\right\rangle, \ldots,\left\langle u_{n}, e_{n}\right\rangle\right)=\left(\sum_{j=1}^{n} w_{j} d_{j}^{\lambda}\right)^{1 / \lambda}
$$

Since $\left(\left\langle u_{1}, a_{1}\right\rangle, \ldots,\left\langle u_{n}, a_{n}\right\rangle\right)$ is a permutation of $\left(\left\langle u_{1}, e_{1}\right\rangle, \ldots,\left\langle u_{n}, e_{n}\right\rangle\right)$, we have $a_{j}=e_{j}$, for all $j$, so

$$
f\left(\left\langle u_{1}, a_{1}\right\rangle, \ldots,\left\langle u_{n}, a_{n}\right\rangle\right)=f\left(\left\langle u_{1}, e_{1}\right\rangle, \ldots,\left\langle u_{n}, e_{n}\right\rangle\right) .
$$

Theorem 3. (Idempotency): Let $f$ be the IGOWA operator. If $a_{i}=a$, for all $a_{i}$, then

$$
f\left(\left\langle u_{1}, a_{1}\right\rangle, \ldots,\left\langle u_{n}, a_{n}\right\rangle\right)=a .
$$

Proof. Since $a_{i}=a$, for all $a_{i}$, we have

$$
f\left(\left\langle u_{1}, a_{1}\right\rangle, \ldots,\left\langle u_{n}, a_{n}\right\rangle\right)=\left(\sum_{j=1}^{n} w_{j} b_{j}^{\lambda}\right)^{1 / \lambda}=\left(\sum_{j=1}^{n} w_{j} a^{\lambda}\right)^{1 / \lambda}=\left(a^{\lambda} \sum_{j=1}^{n} w_{j}\right)^{1 / \lambda} .
$$


Since $\sum_{j=1}^{n} w_{j}=1$, we get

$$
f\left(\left\langle u_{1}, a_{1}\right\rangle, \ldots,\left\langle u_{n}, a_{n}\right\rangle\right)=a .
$$

Theorem 4. (Bounded): Let $f$ be the IGOWA operator. Then

$$
\min \left\{a_{i}\right\} \leqslant f\left(\left\langle u_{1}, a_{1}\right\rangle, \ldots,\left\langle u_{n}, a_{n}\right\rangle\right) \leqslant \max \left\{a_{i}\right\}
$$

Proof. Let $\max \left\{a_{i}\right\}=c$, and $\min \left\{a_{i}\right\}=d$. Then

$$
f\left(\left\langle u_{1}, a_{1}\right\rangle, \ldots,\left\langle u_{n}, a_{n}\right\rangle\right)=\left(\sum_{j=1}^{n} w_{j} b_{j}^{\lambda}\right)^{1 / \lambda} \leqslant\left(\sum_{j=1}^{n} w_{j} c^{\lambda}\right)^{1 / \lambda}=\left(c^{\lambda} \sum_{j=1}^{n} w_{j}\right)^{1 / \lambda}
$$

and

$$
f\left(\left\langle u_{1}, a_{1}\right\rangle, \ldots,\left\langle u_{n}, a_{n}\right\rangle\right)=\left(\sum_{j=1}^{n} w_{j} b_{j}^{\lambda}\right)^{1 / \lambda} \geqslant\left(\sum_{j=1}^{n} w_{j} d^{\lambda}\right)^{1 / \lambda}=\left(d^{\lambda} \sum_{j=1}^{n} w_{j}\right)^{1 / \lambda} .
$$

Since $\sum_{j=1}^{n} w_{j}=1$, we get

$$
f\left(\left\langle u_{1}, a_{1}\right\rangle, \ldots,\left\langle u_{n}, a_{n}\right\rangle\right) \leqslant c
$$

and

$$
f\left(\left\langle u_{1}, a_{1}\right\rangle, \ldots,\left\langle u_{n}, a_{n}\right\rangle\right) \geqslant d .
$$

Therefore,

$$
\min \left\{a_{i}\right\} \leqslant f\left(\left\langle u_{1}, a_{1}\right\rangle, \ldots,\left\langle u_{n}, a_{n}\right\rangle\right) \leqslant \max \left\{a_{i}\right\} .
$$

An interesting issue in analyzing induced aggregation operators is the problem of ties in the reordering step. To solve this problem, we recommend following the method developed by Yager and Filev [43] where they replace each argument of the tied IOWA pair by its average. For the IGOWA operator, instead of using the arithmetic mean, we replace each argument of the tied IGOWA pair by its generalized mean depending on the parameter of $\lambda$.

As explained in [43] for the IOWA operator, we should note that the values used for the order-inducing variables of the IGOWA operator, can be drawn from any space that has a linear ordering. Thus, it is possible to use different kinds of attributes for the order-inducing variables; in particular, we can mix numbers with words in the aggregations [43]. For the IGOWA operator, this would mean that we are ordering numerical arguments by linguistic order-inducing variables. Note that in some situations it is possible to use the implicit lexicographic ordering associated with words, i.e. the ordering of words in the dictionary [43].

The IGOWA operator is a generalization of the IOWA operator. Therefore, the IGOWA operator is applicable to all the situations already discussed for the IOWA operator. For example, we could use it for modeling the nearest neighbour rule [43], for model building [43] and for the aggregation of complex objects [35]. Other potential applications could be developed for decision-making, group decision-making, business decisions, statistics, etc. In this paper, we develop an application for financial decision-making.

\section{Families of IGOWA operators}

In this section, we consider different types of IGOWA operators. We distinguish between two main classes. The first class focuses on the weighting vector $W$, and the second class on the parameter $\lambda$. In Table 1 , we present the main families of IGOWA operators that we consider in this paper.

The main advantage of using these families is that they can be very useful for the decision-maker in some specific situations. However, each family is just one particular case. Therefore, they can only be used in some particular cases, but they cannot be seen as a general model that can be used in all possible frameworks. Thus, the best way to assess all these particular cases is by using a general formulation such as the IGOWA operator that includes them all. Note that the particular case to be used will depend on the interests of the decision-maker in the specific problem at hand.

\subsection{Analyzing the weighting vector $W$}

By choosing a different manifestation of the weighting vector in the IGOWA operator, we are able to obtain different types of aggregation operators. For example, we can obtain the maximum, the minimum, the generalized mean, the weighted generalized mean and the GOWA operator. Note that these results can be obtained both for the DIGOWA and the AIGOWA operator. 
Table 1

Families of IGOWA operators.

\begin{tabular}{ll}
\hline Weighting vector W & Parameter $\lambda$ \\
\hline - Maximum and minimum & • IOWA operator \\
- Generalized mean and weighted generalized mean & • IOWG operator \\
- GOWA operator & - IOWHA operator \\
- Window-IGOWA & - EWQA operator \\
- Olympic-IGOWA & \\
- E-Z IGOWA & \\
- Generalized median and weighted generalized median & \\
- S-IGOWA (orlike, andlike and generalized) & \\
- BADD-IGOWA (Dependent - IGOWA) & \\
- BUM function - IGOWA & \\
- Centered IGOWA and Gaussian-IGOWA & \\
- Etc. &
\end{tabular}

Remark 1. The maximum is obtained by setting $w_{p}=1$ and $w_{j}=0$, for all $j \neq p$, and $u_{p}=\operatorname{Max}\left\{a_{i}\right\}$, and the minimum by setting $w_{p}=1$ and $w_{j}=0$, for all $j \neq p$, and $u_{p}=\operatorname{Min}\left\{a_{i}\right\}$. More generally, if $w_{k}=1$ and $w_{j}=0$, for all $j \neq k$, we get for any $\lambda$, IGOWA( $\left\langle u_{1}\right.$, $\left.\left.a_{1}\right\rangle, \ldots,\left\langle u_{n}, a_{n}\right\rangle\right)=b_{k}$, where the $b_{k}$ as usual, are the $a_{i}$ values ordered by their associated $u_{i}$ values. The generalized mean is found by setting $w_{j}=1 / n$, for all $a_{i}$ and the weighted generalized mean is obtained if $u_{i}>u_{i+1}$, for all $i$. Finally, we recover the GOWA operator if the ordered positions of $u_{i}$ are the same as the ordered positions of the $a_{i}$.

Remark 2. Other families of IGOWA operators can be constructed by choosing a different weighting vector. For example, when $w_{j}=1 / m$ for $k \leqslant j \leqslant k+m-1$ and $w_{j}=0$ for $j>k+m$ and $j<k$, we obtain the window-IGOWA operator that it is based on the window-OWA operator [31]. Note that $k$ and $m$ must be positive integers such that $k+m$ $1 \leqslant n$. Note also that if $m=k=1$, and the initial position of the highest $u_{i}$ is also the initial position of the highest $a_{i}$, then the window-IGOWA becomes the maximum. If $m=1, k=n$, and the initial position of the lowest $u_{i}$ is also the initial position of the lowest $a_{i}$, then, it becomes the minimum. Finally, if $m=n$ and $k=1$, it becomes the generalized mean.

Example 2. (window-IGOWA). Assume a weighting vector of dimension $7(n=7)$. If $k=2$ and $m=4$, then the weighting vector to be used in the aggregation is $W=(0,0.25,0.25,0.25,0.25,0,0)$.

Remark 3. If $w_{1}=w_{n}=0$, and for all others $w_{j}=1 /(n-2)$, we use the olympic induced generalized average, which is based on the olympic average [33]. Note that if $n=3$ or $n=4$, the olympic induced generalized average becomes the IGOWA median, and if $m=n-2$ and $k=2$, the window-IGOWA becomes the olympic induced generalized average. Note also that the olympic induced generalized average becomes the olympic generalized average if $w_{p}=w_{q}=0$, such that $u_{p}=\operatorname{Max}_{i}\left\{a_{i}\right\}$ and $u_{q}=$ Mi$n_{i}\left\{a_{i}\right\}$, and for all others $w_{j}=1 /(n-2)$.

Example 3. (Olympic-IGOWA). Assume a weighting vector of dimension $7(n=7)$. Then the weighting vector to be used in the aggregation is $W=(0,0.2,0.2,0.2,0.2,0.2,0)$.

Remark 4. Another type of aggregation is the E-Z IGOWA weights, which are based on the E-Z OWA weights [36]. In this case, we should distinguish between two classes. In the first class, we assign $w_{j}=(1 / k)$ for $j=1$ to $k$ and $w_{j}=0$ for $j>k$, and in the second class, we assign $w_{j}=0$ for $j=1$ to $n-k$ and $w_{j}=(1 / k)$ for $j=n-k+1$ to $n$. Note that the E-Z IGOWA weights become the E-Z GOWA weights in the first class if the ordered positions of the $u_{i}$ are the same as those of the $a_{i}$, for $i$ between 1 and $k$, and for the second class, if the ordered positions of the $u_{i}$ are the same as those of the $a_{i}$, for $i$ between $n-k+1$ and $n$.

Example 4. ( $E-Z$ IGOWA). Assume that $k=4$ and $n=7$. For the first class, the weighting vector is $W=(0.25,0.25,0.25,0.25,0$, $0,0)$ and for the second class, $W=(0,0,0,0.25,0.25,0.25,0.25)$.

Remark 5. The generalized median and the weighted generalized median [32] can also be constructed as induced aggregation operators. For the IGOWA median, if $n$ is odd we assign $w_{(n+1) / 2}=1$ and $w_{j}=0$ for all others, which selects the argument $a_{i}$ with the $[(n+1) / 2]$ th largest $u_{i}$ value. If $n$ is even, we assign, for example, $w_{n / 2}=w_{(n / 2)+1}=0.5$, which selects the arguments with the $(n / 2)$ th and $[(n / 2)+1]$ th largest $u_{i}$ values. For the weighted IGOWA median, we select the argument $a_{i}$ that has the $k$ th largest inducing variable $u_{i}$, such that the sum of the weights from 1 to $k$ is equal or higher than 0.5 and the sum of the weights from 1 to $k-1$ is less than 0.5 . Note that if the ordered positions of the $u_{i}$ are the same as the ordered positions of the $a_{i}$, then the IGOWA median and the weighted IGOWA median reduce to the GOWA median and the weighted GOWA median, respectively. 
Example 5. (Median-IGOWA). Assume $n=7$. Then the weighting vector to be used is: $W=(0,0,0,1,0,0,0)$.

Remark 6.1. Another interesting family is the S-IGOWA operator, based on the S-OWA operator [31,42]. It can be divided in three classes, the "orlike", the "andlike" and the generalized S-IGOWA operator. The "orlike" S-IGOWA operator is formed when $w_{p}=(1 / n)(1-\alpha)+\alpha, u_{p}=\operatorname{Max}\left\{a_{i}\right\}$, and $w_{j}=(1 / n)(1-\alpha)$ for all $j \neq p$ with $\alpha \in[0,1]$. Note that if $\alpha=0$, we get the arithmetic mean, and if $\alpha=1$, the maximum. The "andlike" S-IGOWA operator is found when $w_{q}=(1 / n)(1-\beta)+\beta, u_{q}=\operatorname{Min}\left\{a_{i}\right\}$, and $w_{j}=(1 / n)(1-\beta)$ for all $j \neq q$ with $\beta \in[0,1]$. In this class, if $\beta=0$ we get the average, and if $\beta=1$, the minimum. Finally, the generalized S-IGOWA operator is obtained when $w_{p}=(1 / n)(1-(\alpha+\beta))+\alpha$, with $u_{p}=\operatorname{Max}\left\{a_{i}\right\} ; w_{q}=(1 / n)(1-(\alpha+\beta))+\beta$, with $u_{q}=\operatorname{Min}\left\{a_{i}\right\}$; and $w_{j}=(1 / n)(1-(\alpha+\beta))$ for all $j \neq p, q$ where $\alpha, \beta \in[0,1]$ and $\alpha+\beta \leqslant 1$. Note that if $\alpha=0$, the generalized SIGOWA operator becomes the "andlike" S-IGOWA operator, and if $\beta=0$, it becomes the "orlike" S-IGOWA operator.

Remark 6.2. Note that it is also possible to consider the usual definition of the S-OWA without using the inducing orders [42]. In this setting, we form another type of S-IGOWA that does not take into account the maximum and the minimum arguments. Instead, it takes into account the arguments in the first and the last positions as defined by the order-inducing variables. In this case, the generalized S-IGOWA operator is formed when $w_{1}=(1 / n)(1-(\alpha+\beta))+\alpha, w_{n}=(1 / n)(1-(\alpha+\beta))+\beta$, and $w_{j}=(1 / n)(1-(\alpha+\beta))$ for $j=2$ to $n-1$ where $\alpha, \beta \in[0,1]$ and $\alpha+\beta \leqslant 1$. Note that if $\alpha=0$, the generalized S-IGOWA becomes the "andlike" S-IGOWA operator and if $\beta=0$, it becomes the "orlike" S-IGOWA operator.

Example 6. (Generalized S-IGOWA): Take the IGOWA pairs in Example 1, and set $\alpha=0.1$ and $\beta=0.3$. Then the weighting vector to be used in the aggregation is $W=(0.45,0.15,0.25,0.15)$. In the context of Remark 6.2 , the weighting vector is $W=(0.25,0.15,0.15,0.45)$.

Remark 7.1. Other families of IGOWA operators could be developed, with the weights depending on the aggregated objects [31]. Note that in these cases, the results obtained with the IGOWA are equal to the ones obtained with the GOWA because the order-inducing variables do not affect the final result. For example, we could develop the BADD-IGOWA operator based on the OWA version developed in $[31,42]$ :

$$
w_{j}=\frac{b_{j}^{\alpha}}{\sum_{j=1}^{n} b_{j}^{\alpha}},
$$

where $\alpha \in(-\infty, \infty)$, and the $b_{j}$ are the arguments $a_{i}$ ordered in decreasing order. Note that the sum of the weights is 1 and $w_{j} \in[0,1]$. Note also that if $\alpha=0$, we get the generalized mean, and if $\alpha=\infty$, the maximum.

Remark 7.2. Another family of IGOWA operators that depend on the aggregated objects is

$$
w_{j}=\frac{\left(1-b_{j}\right)^{\alpha}}{\sum_{j=1}^{n}\left(1-b_{j}\right)^{\alpha}},
$$

where $\alpha \in(-\infty, \infty)$, and the $b_{j}$ are the arguments $a_{i}$ ordered in decreasing order. Note that in this case if $\alpha=0$, we also get the generalized mean and if $\alpha=\infty$, the minimum.

Remark 7.3. A third family of IGOWA operators that depend on the aggregated objects is

$$
w_{j}=\frac{\left(1 / b_{j}\right)^{\alpha}}{u m_{j=1}^{n}\left(1 / b_{j}\right)^{\alpha}},
$$

where $\alpha \in(-\infty, \infty)$, and the $b_{j}$ are the arguments $a_{i}$ in decreasing order. In this case, we also get the generalized mean if $\alpha=0$. If $\alpha=1$, we obtain the harmonic mean and if $\alpha=\infty$, the minimum.

Example 7. (BADD-IGOWA): Taking the IGOWA pairs from Example 1, and $\alpha=1$, the weighting vector obtained is $W=(0.1379,01724,0.4137,0.2758)$.

Remark 8. A very useful approach to obtain the weights that is also applicable for the IGOWA operator is the functional method introduced by Yager [33] for the OWA operator. This approach can be summarized as follows. Let $f$ be a function $f:[0,1] \rightarrow[0,1]$ such that $f(0)=f(1)$ and $f(x) \geqslant f(y)$ for $x>y$. We call this function a basic unit interval monotonic function (BUM). Using this BUM function we obtain the IGOWA weights $w_{j}$ for $j=1$ to $n$ as

$$
w_{j}=f\left(\frac{j}{n}\right)-f\left(\frac{j-1}{n}\right) .
$$

It can be easily shown that using this method, the $w_{j}$ satisfy the conditions that the sum of the weights is 1 and $w_{j} \in[0,1]$.

Example 8. (BUM function): Take $f(x)=x^{2}$ and $n=5$. In this case, the weighting vector to be used is $W=(0.04,0.12,0.2$, $0.28,0.36$ ). 
Remark 9. Another family of aggregation operators that could be used in the IGOWA operator is the centered aggregation operators, which were suggested by Yager [39] for the OWA operator. Following the same methodology, we could say that an IGOWA operator is a centered aggregation operator if it is symmetric, strongly decaying and inclusive. It is symmetric if $w_{j}=w_{j+n-1}$. It is strongly decaying if $i<j \leqslant(n+1) / 2$ then $w_{i}<w_{j}$ and when $i>j \geqslant(n+1) / 2$ then $w_{i}<w_{j}$. It is inclusive if all the $w_{j}>0$. Note that it is possible to consider a softening of the second condition by using $w_{i} \leqslant w_{j}$ instead of $w_{i}<w_{j}$. We shall refer to this as the softly decaying centered IGOWA operator. Note that the generalized mean is an example of this particular case of the centered IGOWA operator. Another generalization of the centered IGOWA operator appears if we remove the third condition. We shall refer to it as a non-inclusive centered IGOWA operator. The IGOWA median is a special case of this operator.

Remark 10. A special type of centered IGOWA operator is the Gaussian-IGOWA weights operator, constructed by analogy with the Gaussian OWA weights suggested by Xu [25]. In order to define it, we have to consider a Gaussian distribution $\eta(\mu, \sigma)$ where

$$
\begin{aligned}
\mu_{n} & =\frac{1}{n} \sum_{j=1}^{n} j=\frac{n+1}{2}, \\
\sigma_{n} & =\sqrt{\frac{1}{n} \sum_{j=1}^{n}\left(j-\mu_{n}\right)^{2}} .
\end{aligned}
$$

Assuming that

$$
\eta(j)=\frac{1}{\sqrt{2 \prod \sigma_{n}}} e^{-\left(j-\mu_{n}\right)^{2} / 2 \sigma_{n}^{2}},
$$

we define the IGOWA weights as

$$
w_{j}=\frac{\eta_{j}}{\sum_{j=\eta}^{n} \eta(j)}=\frac{e^{-\left(j-\mu_{n}\right)^{2} / 2 \sigma_{n}^{2}}}{\sum_{j=1}^{n} e^{-\left(j-\mu_{n}\right)^{2} / 2 \sigma_{n}^{2}}} .
$$

Note that the sum of the weights is 1 and $w_{j} \in[0,1]$.

Example 9. (Gaussian-IGOWA): Set $n=5$. Applying the previous equations, we get the following weighting vector: $W=(0.1117,0.2364,0.3036,0.2364,0.1117)$. As we can see, it is a centered aggregation operator because it satisfies the conditions in Remark 9.

Remark 11. Other weighting vectors could also be used to construct other families of IGOWA operators, by analogy with the other families of OWA operators, e.g., those in $[1,2,16,17,21-25,40]$.

\subsection{Analyzing the parameter $\lambda$}

If we analyze the possible values of the parameter $\lambda$ in the IGOWA operator, we obtain another group of particular cases, including the usual IOWA operator, the induced OWG (IOWG) operator [7,28], the induced OWHA (IOWHA) operator and the induced OWQA (IOWQA) operator.

Remark 12. When $\lambda=1$, the IGOWA operator becomes the IOWA operator:

$$
\operatorname{IGOWA}\left(\left\langle u_{1}, a_{1}\right\rangle,\left\langle u_{2}, a_{2}\right\rangle, \ldots,\left\langle u_{n}, a_{n}\right\rangle\right)=\sum_{j=1}^{n} w_{j} b_{j} .
$$

We can generalize the reordering direction and distinguish between the descending IOWA (DIOWA) operator (with the arguments reordered in descending order) and the ascending IOWA (AIOWA) operator (with the arguments reordered in ascending order). Note that the distinction between descending and ascending orders is also applicable to the IOWG, the IOWHA and the IOWQA operator. An example of the IOWA operator was presented after Definition 5.

Remark 13. When $\lambda=0$, the IGOWA operator becomes the IOWG operator:

$$
\operatorname{IGOWA}\left(\left\langle u_{1}, a_{1}\right\rangle,\left\langle u_{2}, a_{2}\right\rangle, \ldots,\left\langle u_{n}, a_{n}\right\rangle\right)=\prod_{j=1}^{n} b_{j}^{w_{j}} .
$$

Example 10. (IOWG): Using the same collection of IGOWA pairs and the same weighting vector as in Example 1, if we take $\lambda=0$ (IOWG), then the aggregation process yields

$$
\text { IGOWA }=20^{0.2} \times 25^{0.2} \times 60^{0.3} \times 40^{0.3}=35.7978 .
$$


Remark 14. When $\lambda=-1$, we form the IOWHA operator:

$$
\operatorname{IGOWA}\left(\left\langle u_{1}, a_{1}\right\rangle,\left\langle u_{2}, a_{2}\right\rangle, \ldots,\left\langle u_{n}, a_{n}\right\rangle\right)=\frac{1}{\sum_{j=1}^{n} \frac{w_{j}}{b_{j}}} .
$$

Example 11. (IOWHA): Using the same information as in Example 1, but now with $\lambda=-1$, the aggregation is

$$
\text { IGOWA }=\frac{1}{\frac{0.2}{20}+\frac{0.2}{25}+\frac{0.3}{60}+\frac{0.3}{40}}=32.7868 .
$$

Remark 15. When $\lambda=2$, we form the IOWQA operator.

$$
\operatorname{IGOWA}\left(\left\langle u_{1}, a_{1}\right\rangle,\left\langle u_{2}, a_{2}\right\rangle, \ldots,\left\langle u_{n}, a_{n}\right\rangle\right)=\left(\sum_{j=1}^{n} w_{j} b_{j}^{2}\right)^{1 / 2}
$$

Example 12. (IOWQA): Assuming the same information as in Example 1, but now with $\lambda=2$, the aggregation becomes

$$
\mathrm{IGOWA}=\left(0.2 \times 20^{2}+0.2 \times 25^{2}+0.3 \times 60^{2}+0.3 \times 40^{2}\right)^{1 / 2}=42.0119 .
$$

Remark 16. Note that other families could be constructed by choosing different values in the parameter $\lambda$. It is also possible to study these families individually. We could then develop analyses for each case similar to the ones carried out in Sections 3 and 4.1 , and study different properties and families of the induced aggregation operators.

\section{Induced Quasi-OWA operators}

As it is explained in [3], a further generalization of the GOWA operator is possible by using quasi-arithmetic means instead of the ordinary means. Following a similar methodology, we can suggest a similar generalization of the IGOWA operator, to obtain the Quasi-IOWA operator. The main advantage of using this operator is that it provides a more complete generalization, including a lot of cases that are not included in the IGOWA operator. It can be defined as follows:

Definition 6. A Quasi-IOWA operator of dimension $n$ is a mapping QIOWA: $R^{n} \rightarrow R$ defined by an associated weighting vector $W$ of dimension $n$ such that the sum of the weights is 1 and $w_{j} \in[0,1]$, and by a strictly monotonic continuous function $g(b)$, as follows:

$$
\operatorname{QIOWA}\left(\left\langle u_{1}, a_{1}\right\rangle,\left\langle u_{2}, a_{2}\right\rangle, \ldots,\left\langle u_{n}, a_{n}\right\rangle\right)=g^{-1}\left(\sum_{j=1}^{n} w_{j} g\left(b_{(j)}\right)\right)
$$

where the $b_{j}$ are the argument values $a_{i}$ of the Quasi-IOWA pairs $\left\langle u_{i}, a_{i}\right\rangle$ ordered in decreasing order of their $u_{i}$, values.

As we can see, the difference between the IGOWA and the Quasi-IOWA, is that we replace $b^{\lambda}$ with a general continuous strictly monotonic function $g(b)$.

In this case also, we can distinguish between descending (Quasi-DIOWA) and ascending (Quasi-AIOWA) orders. The weights of these operators are related by $w_{j}=w_{n+1-j}^{*}$, where $w_{j}$ is the $j$ th weight of the Quasi-DIOWA (or Quasi-IOWA) operator and $w_{n+1-j}^{*}$ the $j$ th weight of the Quasi-AIOWA operator.

Note also that all the properties and particular cases of the IGOWA operator also apply in this generalization. As such, the Quasi-IOWA operator is monotonic, bounded, idempotent and commutative. The problem of ties is solved by replacing the tied arguments by the quasi-arithmetic mean. And it is possible to analyze different families of Quasi-IOWA operators such as the olympic-Quasi-IOWA, the S-Quasi-IOWA, the IOWA itself, the IOWQA, etc.

The Quasi-IOWA operator also includes a lot of other particular cases that are not included in the IGOWA operator. For example, we could mention the trigonometric IOWA operator, the exponential IOWA operator and the radical IOWA operator.

The trigonometric IOWA is found when $g_{1}(t)=\sin ((\pi / 2) t), g_{2}(t)=\cos ((\pi / 2) t)$ and $g_{3}(t)=\tan ((\pi / 2) t)$ are the generating functions. Thus, the trigonometric IOWA functions are:

$$
\begin{aligned}
& \operatorname{QIOWA}\left(\left\langle u_{1}, a_{1}\right\rangle,\left\langle u_{2}, a_{2}\right\rangle, \ldots,\left\langle u_{n}, a_{n}\right\rangle\right)=\frac{2}{\pi} \arcsin \left(\sum_{j=1}^{n} w_{j} \sin \left(\frac{\pi}{2} b_{j}\right)\right), \\
& \operatorname{QIOWA}\left(\left\langle u_{1}, a_{1}\right\rangle,\left\langle u_{2}, a_{2}\right\rangle, \ldots,\left\langle u_{n}, a_{n}\right\rangle\right)=\frac{2}{\pi} \arccos \left(\sum_{j=1}^{n} w_{j} \cos \left(\frac{\pi}{2} b_{j}\right)\right),
\end{aligned}
$$

and

$$
\operatorname{QIOWA}\left(\left\langle u_{1}, a_{1}\right\rangle,\left\langle u_{2}, a_{2}\right\rangle, \ldots,\left\langle u_{n}, a_{n}\right\rangle\right)=\frac{2}{\pi} \arctan \left(\sum_{j=1}^{n} w_{j} \tan \left(\frac{\pi}{2} b_{j}\right)\right) .
$$


Table 2

Payoff matrix

\begin{tabular}{lllll}
\hline & $S_{1}$ & $S_{2}$ & $S_{3}$ & $S_{4}$ \\
\hline$A_{1}$ & 80 & 50 & 70 & 40 \\
$A_{2}$ & 60 & 30 & 80 & 80 \\
$A_{3}$ & 70 & 50 & 20 & 70 \\
$A_{4}$ & 50 & 40 & 60 & 90 \\
$A_{5}$ & 20 & 50 & 50 & 80 \\
\hline
\end{tabular}

Table 3

Inducing variables.

\begin{tabular}{lllll}
\hline & $S_{1}$ & $S_{2}$ & $S_{3}$ & $S_{5}$ \\
\hline$A_{1}$ & 17 & 10 & 15 & 22 \\
$A_{2}$ & 15 & 20 & 22 & 20 \\
$A_{3}$ & 24 & 18 & 20 & 22 \\
$A_{4}$ & 16 & 19 & 21 & 25 \\
$A_{5}$ & 18 & 12 & 26 & 25 \\
\hline
\end{tabular}

Table 4

Aggregated results 1 .

\begin{tabular}{lllllll}
\hline & AM & WA & OWA & AOWA & AIOWA \\
\hline$A_{1}$ & 60 & 58 & 56 & 64 & 61 \\
$A_{2}$ & 58 & 56 & 53 & 63 & 54 \\
$A_{3}$ & 60 & 62 & 53 & 67 & 62 \\
$A_{4}$ & 56 & 58 & 53 & 59 & 52 \\
$A_{5}$ & 56 & 62 & 50 & 52 & 58 \\
\hline
\end{tabular}

Table 5

Aggregated results 2 .

\begin{tabular}{llllll}
\hline & QA & IOWQA & IOWG & Step & Olympic \\
\hline$A_{1}$ & 56.92 & 62.36 & 59.58 & 80 & 70 \\
$A_{2}$ & 61.48 & 57.44 & 50.41 & 80 & 70 \\
$A_{3}$ & 64.49 & 66.93 & 54.92 & 50 & 20 \\
$A_{4}$ & 56.92 & 54.77 & 53.19 & 60 & 80.6 \\
$A_{5}$ & 60.33 & 60.33 & 50.23 & 80 & 80 \\
\hline
\end{tabular}

Table 6

Ordering of the investments.

\begin{tabular}{|c|c|c|c|}
\hline & Ordering & & Ordering \\
\hline AM & & $\mathrm{QA}$ & \\
\hline WA & $A_{3}$ & IOWQA & \\
\hline OWA & & IOWG & \\
\hline AOWA & & Step-IOWA & \\
\hline IOWA & & Median-IOWA & \\
\hline AIOWA & & Olympic-IOWA & \\
\hline
\end{tabular}


The exponential IOWA is obtained by setting $g(t)=\gamma^{t}$, if $\gamma \neq 1$, and $g(t)=t$, if $\gamma=1$. Then, the exponential IOWA operator is: $\log _{\gamma}\left(\sum_{j=1}^{n} w_{j} \gamma^{b_{j}}\right)$, if $\gamma \neq 1$, and is equal to the ordinary IOWA if $\gamma=1$.

The radical loWA is found by taking as the generating function $g(t)=\gamma^{1 / t}$, for some parameter $\gamma>0, \gamma \neq 1$. Thus, the radical IOWA operator is

$$
\operatorname{QIOWA}\left(\left\langle u_{1}, a_{1}\right\rangle,\left\langle u_{2}, a_{2}\right\rangle, \ldots,\left\langle u_{n}, a_{n}\right\rangle\right)=\left(\log _{\gamma}\left(\sum_{j=1}^{n} w_{j} \gamma^{1 / b_{j}}\right)\right)^{-1} .
$$

Finally, note that it is also possible to study the properties and different particular cases of all these operators by analogy with Sections 3 and 4.1 .

\section{Numerical example}

In the following, we present an illustrative example of the new approach in a decision-making problem. We study an investment selection problem where an investor is looking for an optimal investment. Note that other decision-making applications could be developed along similar lines, such as the selection of financial products [19], human resource management, strategic decision-making, and product management.

We will analyze different particular cases of the IGOWA operator such as the AM, the WA, the OWA, the AOWA, the IOWA, the AIOWA, the QM, the IOWG, the IOWQA, the step-IOWA $(k=2)$, the median-IOWA and the olympic-IOWA. Note that with this analysis, we obtain "optimal" choices that depend on the aggregation operator used. Then, we will see that each aggregation operator may lead to different results and decisions. Obviously, the question, as in other decision-making problems, is the selection of the aggregation operator. By now, the answer we can give is that each decision-maker will select one or more aggregation operators that reflect his interests. Furthermore, depending on the aggregation operator used, his decisions will be different. The main advantage of the IGOWA is that it includes a wide range of particular cases, reflecting different potential factors to be considered in the decision-making problem. Thus, the decision-maker is able to consider a lot of possibilities and select the aggregation operator that is in closest accordance with his interests.

Assume that an investor wants to invest some money in an enterprise in order to get the highest possible profits. Initially, he considers five possible alternatives.

- $A_{1}$ is a computer company.

- $A_{2}$ is a chemical company.

- $A_{3}$ is a food company.

- $A_{4}$ is a car company.

- $A_{5}$ is a TV company.

In order to evaluate these investments, the investor has brought together a group of experts. This group considers that the key factor is the economic environment in the global economy. After careful analysis, they consider five possible situations for the economic environment: $S_{1}=$ negative growth rate, $S_{2}=$ growth rate near $0, S_{3}=$ low growth rate, $S_{4}=$ medium growth rate, $S_{5}=$ high growth rate. The expected results of the investment, depending on the situation $S_{i}$ that occurs and the alternative $A_{k}$ that the investor chooses, are shown in Table 2.

In this problem, the experts assume the following weighting vector: $W=(0.1,0.2,0.2,0.2,0.3)$. Due to the fact that the attitudinal character is very complex because it involves the opinion of different members of the board of directors, the experts use order-inducing variables to represent it. The results are shown in Table 3.

With this information, we can aggregate the expected results for each state of nature in order to make a decision. In Tables 4 and 5, we present different results obtained using different types of IGOWA operators.

If we establish an ordering of the alternatives, a typical situation if we want to consider more than one alternative, then we get the results shown in Table 6. Note that the first alternative in each ordering is the optimal choice.

As we can see, depending on the aggregation operator used, the ordering of the investments may be different. Therefore, the decision about which investment or investments to select may be also different.

\section{Conclusions}

In this paper, we have presented the IGOWA operator. It has the main characteristics of the GOWA and the IOWA operator. That is to say, it uses generalized means and order-inducing variables in the reordering process of the OWA operator. Therefore, it can be seen as a generalization of the IOWA operator to use generalized means or as an extension of the GOWA operator to use order-inducing variables in the reordering of the arguments. With the IGOWA operator, we have been able to generalize a wide range of OWA operators, including all the cases of the IOWA and the GOWA operator, as well as many others such as the IOWG and the IOWQA operators. Moreover, we have further generalized the IGOWA operator by using quasiarithmetic means. We thus obtained the Quasi-IOWA operator, which is a wider generalization that includes the IGOWA operator along with many other useful operators as special cases. 
We also presented a numerical example of the new approach, in order to see the applicability of the IGOWA operator in a financial decision-making problem. The main advantage of this aggregation operator is that it includes a wide range of special cases; depending on the aggregation used, the results and decisions may be different. Thus, by using the IGOWA operator, we are able to assess all these situations in the same framework.

In future research, we expect to develop further extensions by adding new characteristics, such as the use of uncertain information (represented in the form of interval numbers, fuzzy numbers, linguistic variables, etc). We will also consider other decision-making problems, such as strategic decision-making and product management.

\section{Acknowledgements}

We would like to thank the Editor-in-Chief, Professor W. Pedrycz, and the anonymous referees for their valuable comments and suggestions, which led to an improved version of the paper.

\section{References}

[1] G.R. Amin, Note on a preemptive goal programming method for aggregating OWA operator weights in group decision making, Information Sciences 177 (2007) 3636-3638.

[2] B.S. Ahn, Preference relation approach for obtaining OWA operator weights, International Journal of Approximate Reasoning 47 (2008) $166-178$.

[3] G. Beliakov, Learning weights in the generalized OWA operators, Fuzzy Optimization and Decision Making 4 (2005) 119-130.

[4] G. Beliakov, A. Pradera, T. Calvo, Aggregation Functions: A Guide for Practitioners, Springer-Verlag, Berlin, 2007.

[5] T. Calvo, G. Mayor, R. Mesiar, Aggregation Operators: New Trends and Applications, Physica-Verlag, New York, 2002.

[6] F. Chiclana, F. Herrera, E. Herrera-Viedma, The ordered weighted geometric operator: properties and application, in: Proceedings of the Eighth International Conference on Information Processing and Management of Uncertainty in Knowledge-Based Systems, Madrid, Spain, 2000, pp. 985991.

[7] F. Chiclana, F. Herrera, E. Herrera-Viedma, S. Alonso, Induced ordered weighted geometric operators and their use in the aggregation of multiplicative preference relations, International Journal of Intelligent Systems 19 (2004) 233-255.

[8] F. Chiclana, E. Herrera-Viedma, F. Herrera, S. Alonso, Some induced ordered weighted averaging operators and their use for solving group decisionmaking problems based on fuzzy preference relations, European Journal of Operational Research 182 (2007) 383-399.

[9] J. Dujmovic, Weighted conjunctive and disjunctive means and their application in system evaluation, Publikacije Elektro-technickog Faculteta Beograd, Serija Mate-matika i Fizika (483) (1974) 147-158.

[10] H. Dyckhoff, W. Pedrycz, Generalized means as a model of compensative connectives, Fuzzy Sets and Systems 14 (1984) $143-154$.

[11] J. Fodor, J.L. Marichal, M. Roubens, Characterization of the ordered weighted averaging operators, IEEE Transactions on Fuzzy Systems 3 (1995) 236240.

[12] E. Herrera-Viedma, S. Alonso, F. Chiclana, F. Herrera, A consensus model for group decision making with incomplete fuzzy preference relations, IEEE Transactions on Fuzzy Systems 15 (2007) 863-877.

[13] E. Herrera-Viedma, F. Chiclana, F. Herrera, S. Alonso, Group decision-making model with incomplete fuzzy preference relations based on additive consistency, IEEE Transactions on Systems, Man and Cybernetics B 37 (2007) 176-189.

[14] Y.C. Hu, J.F. Tsai, Fusing fuzzy association rule-based classifiers using Sugeno integrals with ordered weighted averaging operators, International Journal of Uncertainty, Fuzziness and Knowledge-Based Systems 15 (2007) 717-735.

[15] N. Karayiannis, Soft learning vector quantization and clustering algorithms based on ordered weighted aggregation operators, IEEE Transactions on Neural Networks 11 (2000) 1093-1105.

[16] X. Liu, The solution equivalence of minimax disparity and minimum variance problems for OWA operators, International Journal of Approximate Reasoning 45 (2007) 68-81.

[17] X. Liu, S. Han, Orness and parameterized RIM quantifier aggregation with OWA operators: a summary, International Journal of Approximate Reasoning 48 (2008) 77-97.

[18] B. Llamazares, Choosing OWA operator weights in the field of social choice, Information Sciences 177 (2007) 4745-4756.

[19] J.M. Merigó, A.M. Gil-Lafuente, Unification point in methods for the selection of financial products, Fuzzy Economic Review 12 (2007) 35-50.

[20] H.B. Mitchell, D.D. Estrakh, A modified OWA operator its use in lossless DPCM image compression, International Journal of Uncertainty, Fuzziness and Knowledge-Based Systems 5 (1997) 429-436.

[21] J.H. Wang, J. Hao, A new version of 2-tuple fuzzy linguistic representation model for computing with words, IEEE Transactions on Fuzzy Systems 14 (2006) 435-445.

[22] Y.M. Wang, Y. Luo, Z. Hua, Aggregating preference rankings using OWA operator weights, Information Sciences 177 (2007) $3356-3363$.

[23] Y.M. Wang, C. Parkan, A preemptive goal programming method for aggregating OWA operator weights in group decision making, Information Sciences 177 (2007) 1867-1877.

[24] J. Wu, C.Y. Liang, Y.Q. Huang, An argument-dependent approach to determining OWA operator weights based on the rule of maximum entropy, International Journal of Intelligent Systems 22 (2007) 209-221.

[25] Z.S. Xu, An overview of methods for determining OWA weights, International Journal of Intelligent Systems 20 (2005) 843-865.

[26] Z.S. Xu, J. Chen, An interactive method for fuzzy multiple attribute group decision making, Information Sciences 177 (2007) $248-263$

[27] Z.S. Xu, Q.L. Da, The ordered weighted geometric averaging operators, International Journal of Intelligent Systems 17 (2002) $709-716$.

[28] Z.S. Xu, Q.L. Da, An overview of operators for aggregating information, International Journal of Intelligent Systems 18 (2003) 953-969.

[29] R.R. Yager, On ordered weighted averaging aggregation operators in multi-criteria decision making, IEEE Transactions on Systems, Man and Cybernetics B 18 (1988) 183-190.

[30] R.R. Yager, On generalized measures of realization in uncertain environments, Theory and Decision 33 (1992) 41-69.

[31] R.R. Yager, Families of OWA operators, Fuzzy Sets and Systems 59 (1993) 125-148.

[32] R.R. Yager, On weighted median aggregation, International Journal of Uncertainty, Fuzziness and Knowledge-Based Systems 2 (1994) 101-113.

[33] R.R. Yager, Quantifier guided aggregation using OWA operators, International Journal of Intelligent Systems 11 (1996) 49-73.

[34] R.R. Yager, The induced fuzzy integral aggregation operator, International Journal of Intelligent Systems 17 (2002) 1049-1065.

[35] R.R. Yager, Induced aggregation operators, Fuzzy Sets and Systems 137 (2003) 59-69.

[36] R.R. Yager, E-Z OWA weights, in: Proceedings of the 10th International Fuzzy Systems Association (IFSA) World Congress, Istanbul, Turkey, 2003, pp. $39-42$.

[37] R.R. Yager, Choquet aggregation using order inducing variables, International Journal of Uncertainty, Fuzziness and Knowledge-Based Systems 12 (2004) 69-88.

[38] R.R. Yager, Generalized OWA aggregation operators, Fuzzy Optimization and Decision Making 3 (2004) 93-107.

[39] R.R. Yager, Centered OWA operators, Soft Computing 11 (2007) 631-639.

[40] R.R. Yager, Using stress functions to obtain OWA operators, IEEE Transactions on Fuzzy Systems 15 (2007) 1122-1129. 
[41] R.R. Yager, Using trapezoids for representing granular objects: applications to learning and OWA aggregation, Information Sciences 178 (2008) 363380.

[42] R.R. Yager, D.P. Filev, Parameterized “andlike" and “orlike” OWA operators, International Journal of General Systems 22 (1994) 297-316.

[43] R.R. Yager, D.P. Filev, Induced ordered weighted averaging operators, IEEE Transactions on Systems, Man and Cybernetics B 29 (1999) $141-150$.

[44] R.R. Yager, J. Kacprzyk, The Ordered Weighted Averaging Operators: Theory and Applications, Kluwer Academic Publishers, Norwell, MA, 1997.

[45] D.Y. Yeh, C.H. Cheng, H.W. Yio, Empirical research of the principal component analysis and ordered weighted averaging integrated evaluation model on software projects, Cybernetics and Systems 38 (2007) 289-303. 